\title{
ON THE NORMS OF $r$-CIRCULANT MATRICES WITH THE HYPER-FIBONACCI AND LUCAS NUMBERS
}

\section{Mustafa BAHŞI AND SÜLEYMAN SOLAK}

Abstract. In this paper, we study norms of circulant matrices $F=\operatorname{Circ}\left(F_{0}^{(k)}, F_{1}^{(k)}, \ldots, F_{n-1}^{(k)}\right)$, $L=\operatorname{Circ}\left(L_{0}^{(k)}, L_{1}^{(k)}, \ldots, L_{n-1}^{(k)}\right)$ and $r$-circulant matrices $F_{r}=\operatorname{Circr}\left(F_{0}^{(k)}, F_{1}^{(k)}, \ldots, F_{n-1}^{(k)}\right), L_{r}=$ $\operatorname{Circr}\left(L_{0}^{(k)}, L_{1}^{(k)}, \ldots, L_{n-1}^{(k)}\right)$, where $F_{n}^{(k)}$ and $L_{n}^{(k)}$ denote the hyper-Fibonacci and hyper-Lucas numbers, respectively.

Mathematics subject classification (2010): 15A60, 15B05, 15B36, 11B39.

Keywords and phrases: Circulant matrix, $r$-circulant matrix, hyper-Fibonacci numbers, hyper-Lucas numbers, Euclidean norm, spectral norm.

\section{REFERENCES}

[1] M. Akbulak And D. BozKuRT, On the norms of Toeplitz, matrices involving Fibonacci and Lucas numbers, Hacettepe Journal of Mathematics and Statistics 37 (2008), pp. 89-95.

[2] M. BAhSi AND S. SolaK, On the circulant matrices with arithmetic sequence, Int. J. Cont. Math. Sciences 5 (25), (2010), pp. 1213-1222.

[3] N.-N. CAO AND F-Z. ZhaO, Some Properties of Hyperfibonacci and Hyperlucas Numbers, Journal of Integer Sequences 13 (2010), Article 10.0.8.

[4] P. J. Davis, Circulant Matrices, Wiley, New York, Chichester, Brisbane, 1979.

[5] A. Dil And I. MezÖ, A symmetric algorithm for hyperharmonic and Fibonacci numbers, Appl. Math. Comp. 206 (2008), pp. 942-951.

[6] R. A. Horn, C. R. Johnson, Matrix Analysis, Cambridge University Press, Cambridge, 1985.

[7] R. A. Horn, C. R. Johnson, Topics in Matrix Analysis, Cambridge University Press, Cambridge, 1991.

[8] A. İPEK, On the spectral norms of circulant matrices with classical Fibonacci and Lucas numbers entries, Appl. Math. Comp. 217 (2011), pp. 6011-6012.

[9] H. Karner, J. Schneid, And C. W. Ueberhuber, Spectral Decomposition of Real Circulant Matrices, Linear Algebra and Its Appl. 367 (2003), pp. 301-311.

[10] E. KILIÇ AND D. TAŞCI, On the generalized order k-Fibonacci and Lucas numbers, Rocky Mountain J. Math. 36 (6) (2006), pp. 1915-1926.

[11] E. G. Kocer, Circulant, negacyclic and semicirculant matrices with the modified Pell, Jacobsthal and Jacobsthal-Lucas numbers, Hacettepe Journal of Mathematics and Statistics 36 (2) (2007), pp. $133-142$.

[12] I. Mezö, Several Generating Functions for Second-Order Recurrence Sequences, Journal of Integer Sequences 12 (2009), Article 09.3.7.

[13] A. A. ÖCAL, N. TUĞLU, AND E. AltinişIK, On the representation of k-generalized Fibonacci and Lucas numbers, Appl. Math. Comp. 170 (2005), pp. 584-596.

[14] S. SHEn, AND J. CEn, On the norms of circulant matrices with the $(k, h)$-Fibonacci and $(k, h)$-Lucas numbers, Int. J. Cont. Math. Sciences 6 (2011), pp. 887-894.

[15] S. SHen, AND J. CEN, On the bounds for the norms of $r$-circulant matrices with the Fibonacci and Lucas numbers, Appl. Math. Comp. 216 (2010), pp. 2891-2897.

[16] S. SOLAK, On the norms of circulant matrices with the Fibonacci and Lucas numbers, Appl. Math. Comp. 160 (2005), pp. 125-132. 
[17] S. SolaK, Erratum to "On the Norms of Circulant Matrices with the Fibonacci and Lucas Numbers, [Appl. Math. Comp. 160, (2005), 125-132], Appl. Math. Comp. 190 (2007), pp. 1855-1856.

[18] S. SolAK AND M. BAHŞI, On the spectral norms of Toeplitz matrices with Fibonacci and Lucas numbers, Hacettepe Journal of Mathematics and Statistics 42 (1) (2013), pp. 15-19.

[19] D. TAŞCI AND E. Kılı̧̇, On the order $-k$ generalized Lucas numbers, Appl. Math. Comp. 155 (2004), pp. 637-641.

[20] Y. YAZLIK AND N. TASKARA, On the norms of an $r$-circulant matrix with the generalized $k$ Horadam numbers, Journal of Inequalities and Applications (2013), 2013:394. 